\title{
The time required to diagnose and treat lung cancer in Delhi, India: an updated experience of a public referral center
}

\author{
Vishal Vashistha 1* (D), Chandrashekhar Choudhari', Avneet Garg², Aditi Gupta², Gopanandan Parthasarathy ${ }^{3}$, \\ Deepali Jain ${ }^{4}$, Karan Madan², Vijay Hadda², G. C. Khilnani², Randeep Guleria² and Anant Mohan²
}

\begin{abstract}
Background: Delays that postpone the evaluation and management of malignancy may lead to considerable morbidity. The primary objective of this study was to assess the time required to diagnose and treat lung cancer at an Indian public referral center that predominantly serves lower-income patients.

Methods: A review of patients diagnosed with lung cancer between January 2008 and December 2016 was completed. We computed the median time intervals and inter-quartile ranges between symptom onset, definitive diagnostic investigation, confirmed histologic diagnosis, and chemotherapy initiation. Median intervals were correlated with baseline demographics and disease characteristics using Kruskal-Wallis test.

Results: One thousand, three hundred and-seventy patients were selected. A majority (94.5\%) with non-small cell lung cancer were diagnosed with advanced disease. After developing symptoms, patients required 101 [56-168] days to undergo a definitive diagnostic study, 107 [60-173] days to confirm a diagnosis, and 126 [85-196.8] days to initiate treatment. Patients who were previously treated for tuberculosis required more time to receive chemotherapy compared to those who were not (187 [134-261.5] days vs. 113 [75-180] days, $p<0.0001)$. A specialty Lung Cancer Clinic was implemented in 2012, and the mean referrals per month increased nearly four-fold $(p<0.0001)$, but the time required to administer treatment was not shortened.

Conclusion: Among lower-income Indian patients, the most prominent delays occur prior to diagnosis. Efforts should be directed toward encouraging physicians to maintain a high index of clinical suspicion and educating patients to report concerning symptoms as early as possible.
\end{abstract}

Keywords: Lung malignancy, Evaluation delays, Management delays, Socioeconomic factors, Developing world, Access to care

\section{Background}

Lung cancer remains the greatest contributor to cancerrelated mortality worldwide $[1,2]$. Patients are often staged with advanced disease at time of diagnosis, thereby limiting curative treatment options. Although low-dose computed tomography is now a validated tool to screen patients and detect lung tumors at earlier stages, preliminary data suggests that screening has yet to be widely implemented, even among populations of higher socioeconomic

\footnotetext{
*Correspondence: vashistha87@gmail.com; vishal.vashistha@duke.edu 'Department of Medicine, Divisions of Hematology, Medical Oncology, and Hematologic Malignancies, Duke University Health System, Durham, NC, USA Full list of author information is available at the end of the article
}

status [3, 4]. As such, for those at higher-risk for lung cancer who have not undergone routine screening, prompt evaluation of new respiratory symptoms is necessary to reduce the likelihood of locally-advanced or metastatic disease [5-7]. Thereafter, the expedited administration of treatment prevents further tumor growth and cancer-related complications. Both public and private healthcare centers have therefore directed efforts toward mitigating delays that unnecessarily postpone the diagnosis and management of lung cancer [8-10].

Previous studies that have examined delays used varying terminology to describe the durations of time required to diagnose and treat lung cancer [11]. Herein, we define a

(C) The Author(s). 2019 Open Access This article is distributed under the terms of the Creative Commons Attribution 4.0 International License (http://creativecommons.org/licenses/by/4.0/), which permits unrestricted use, distribution, and 
patient's evaluation duration to be the time required to complete the necessary steps to obtain a definitive diagnosis. In addition, we define a patient's treatment duration to be the time required to design a treatment plan and administer accordingly. Together, evaluation and treatment durations comprise the total duration of time required to initiate treatment for a patient following the initial onset of symptoms.

An array of factors contributes to prolonged evaluation and treatment durations. Common examples may include limited accessibility to healthcare services among patients and reliance on incorrect diagnoses by providers. Consequently, lower-income patients, particularly in the developing world, likely face the greatest barriers to efficiently undergo evaluation and treatment for their underlying cancers. Our group previously reported the experience of patients receiving care at the All India Institute of Medical Sciences (AIIMS), a tertiary referral center predominantly serving the lower-income population of Delhi, India and the National Capital Region [12]. Between 2002 and 2008, patients required approximately 6 months to receive treatment for lung cancer after initially developing symptoms. In an attempt to both curtail the delays that postpone the management of lung cancer and develop a consolidated center of excellence, the AIIMS Department of Pulmonary Medicine and Sleep Disorders created a formal lung cancer clinic (LCC) specializing in lung cancer care in January 2012. The LCC is uniquely structured to enable medical oncologists, pulmonary specialists, radiation oncologists, and thoracic surgeons to interact with patients simultaneously and to design diagnostic and treatment plans in a collaborative fashion.

As the diagnostic procedures and treatments for lung cancer have advanced over the past decade, a contemporary assessment of the time required to diagnose and treat, specifically among those served in regions of lesser resources, is warranted. Furthermore, the utility of a multidisciplinary LCC at a major referral center should be examined to potentially encourage other large public hospitals to institute a similar collective approach. We have now completed a comprehensive, updated review of our center's experience between January 2008 and December 2016. We report the time to complete each necessary step to diagnose lung cancer and to administer treatment among patients served by a public facility in Delhi, India. We further correlated evaluation and treatment durations with baseline demographics, tumor characteristics, and the development of a specialty LCC in 2012.

\section{Methods}

Data was accessed and collected from medical records within The AIIMS Department of Pulmonary Medicine and Sleep Disorders. This database includes patients who were referred to a lung cancer specialist by providers within the health system or by physicians from external practices. All patients were required to have received a diagnosis of lung cancer based upon cytology or histology prior to referral. Each tissue specimens that was externally rendered a diagnosis of lung cancer were re-reviewed by the thoracic oncology group within the AIIMS Department of Pathology. In case the review was inconclusive, repeat tissue sampling was conducted.

During the initial visit with a lung cancer specialist, each patient was asked to approximate the duration of his or her symptoms prior to presenting to any physician and at the time of referral to our center. Previous medical data, including relevant reports from imaging and procedures, films of conducted imaging, histology slides, and clinic notes authored by other physicians, were sought and reviewed. The dates from all reports and encounters were documented and secured within the medical record for each patient.

\section{Selection criteria}

All adult patients who received a confirmed diagnosis of lung cancer between January 1, 2008 and December 31, 2016 were included. Patients were not excluded based upon procedural modality to obtain a definitive diagnosis or histologic morphology of cancer.

\section{Data collection}

This study was formally approved by the AIIMS Institutional Ethics Committee. The medical records of all included patients were reviewed. Baseline demographics, tumor characteristics, and initial symptoms upon presentation were documented for each patient.

Evaluation durations were defined as the time required to complete the necessary steps to diagnose lung cancer. Treatment durations were defined as the time required to administer chemotherapy following diagnosis. The typical stepwise paradigm for patients undergoing evaluation and treatment is conveyed in Fig. 1.

In regard to evaluation durations, the dates of definitive biopsy, diagnosis rendered by pathology, and first visit with an in-house specialist were collected from the medical records. The subjective duration of symptoms reported by each patient at the time of initial presentation with an in-house specialist was also documented.

In regard to treatment durations, the dates of chemotherapy initiation were documented. Given that a vast majority of our patients were diagnosed with advanced disease, we completed our analyses of treatment durations by only including patients who underwent medical management rather than those who underwent surgery, concurrent chemoradiation, or any other sequential forms of combined modality treatment. Medical management encompasses both traditional cytotoxic chemotherapy and targeted therapies, namely first-generation tyrosine kinase inhibitors. We use the term "chemotherapy" to include both treatments. 


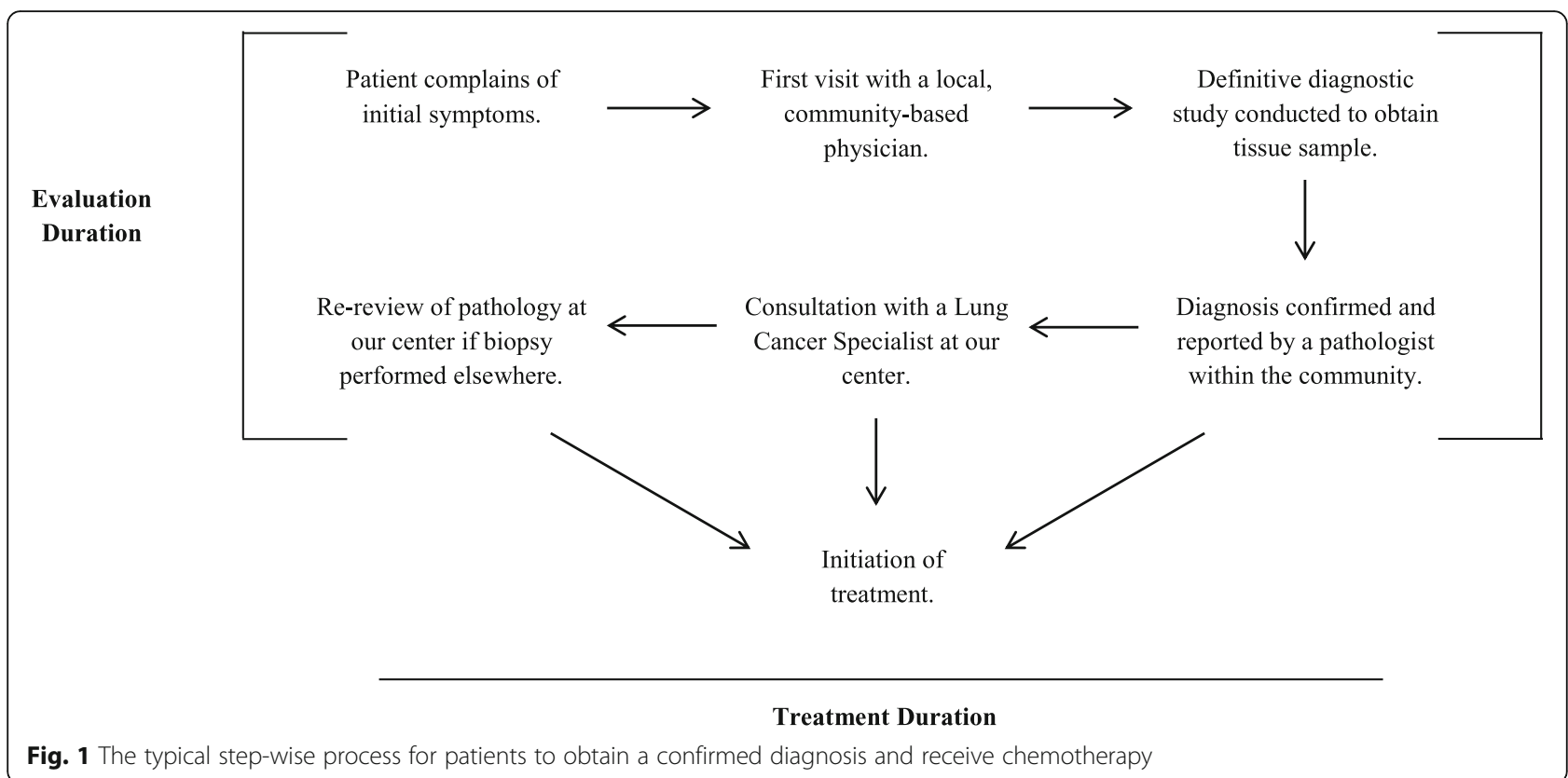

\section{Data analysis}

To determine evaluation durations, the date of symptom onset was approximated for each patient. This was completed by subtracting the reported duration of symptoms from the date of referral to our department. Symptoms that were reported to extend more than 90 days were rounded to the nearest 30-day interval. The durations between dates of symptom onset, definitive test, and histologic diagnosis were calculated for each patient. Outliers who required more than 360 days to receive a confirmed diagnosis of lung cancer were excluded.

To determine treatment durations, the time intervals between the dates of definitive diagnostic test, confirmed diagnosis, first evaluation by an AIIMS specialist, and chemotherapy initiation were calculated for each patient. The approximate date of symptom onset was also used to compute the time interval between the initial development of symptoms and the administration of chemotherapy, thereby providing the total duration of time required to evaluate and treat lung cancer. For total durations, outliers who required more than 720 days to receive systemic treatment were excluded.

Analyses were performed using JMP statistical software [13]. As detailed in previous evaluations of diagnostic and management delays, intervals of time were reported as median days with inter-quartile ranges [14-16]. Univariate analyses were conducted to assess associations between baseline variables and median durations. Given that a specialty LCC was created as a subdivision within the AIIMS Department of Pulmonary Medicine and Sleep Disorders in January 2012, we further compared evaluation, treatment, and total durations prior to January 2012 and thereafter. Comparisons of medians were conducted using
Kruskal-Wallis test while $p<0.05$ was considered significant unless a Bonferroni correction was required to simultaneously analyze three or more durations.

\section{Results}

In total, 1370 patients were included for analysis. Of these, 1140 (83.2\%) were men, and the mean (SD) age was 57.8 (11.0) years. Of the 587 patients who reported their income, 168 (28.6\%) lived below the World Bank poverty line by earning less than $\$ 1.90$ US daily per capita [17]. The baseline demographics and disease characteristics among the included patients are summarized in Table 1. Among all, 249 (18.2\%) patients were evaluated before the development of a specialty LCC at AIIMS in January 2012 and $1120(81.8 \%)$ were evaluated thereafter. The most commonly reported symptoms upon presentation to our center included cough (85.1\%), shortness of breath (73.3\%), and loss of appetite (73.0\%). The least commonly reported symptoms included wheezing (4.9\%), SVC syndrome (5.9\%), and fever (27.7\%).

\section{Interval durations}

The computed evaluation and treatment durations are conveyed in Table 2. Overall, patients required 101 [56168] days to undergo a definitive diagnostic study, 107 [60-173] days to obtain a diagnosis, and 126 [85-196.8] days to receive treatment after initially developing symptoms. Following the creation of a specialty LCC in January 2012, paradoxical increases in time were observed between the following: definitive diagnostic study and confirmed diagnosis $(p<0.0001)$, diagnostic study and chemotherapy initiation $(p=0.028)$, confirmed diagnosis and chemotherapy initiation $(p=0.018)$, and first 
Table 1 Patient demographics

\begin{tabular}{|c|c|}
\hline Age (1369 Responses): & $57.8+/-11.0$ \\
\hline \multicolumn{2}{|l|}{$\operatorname{Sex}(n=1370)$} \\
\hline Male: & $1140(83.2 \%)$ \\
\hline Female: & $230(16.8 \%)$ \\
\hline \multicolumn{2}{|l|}{ Religion $(n=787)$ : } \\
\hline Hindu: & $653(83.0 \%)$ \\
\hline Muslim: & $126(16.0 \%)$ \\
\hline Sikh: & $6(0.8 \%)$ \\
\hline Christian: & $2(0.3 \%)$ \\
\hline \multicolumn{2}{|l|}{ Education $(n=933)$ : } \\
\hline Illiterate: & $232(24.9 \%)$ \\
\hline Primary: & $233(25.0 \%)$ \\
\hline Secondary: & $256(27.4 \%)$ \\
\hline Higher Secondary: & $106(11.4 \%)$ \\
\hline Graduate: & $76(8.1 \%)$ \\
\hline Post-Graduate: & $30(3.2 \%)$ \\
\hline Income per Capita $(n=587)^{\mathrm{a}}$ : & $6000[0-100,000$ \\
\hline \multicolumn{2}{|l|}{ Smoking Status $(n=1273)$ : } \\
\hline Never: & $288(22.6 \%)$ \\
\hline Current: & $534(42.0 \%)$ \\
\hline Reformed: & $451(35.4 \%)$ \\
\hline Smoking Index $(n=1093)^{\mathrm{b}}$ : & $265[0.25-3500]$ \\
\hline \multicolumn{2}{|l|}{ ECOG Performance Status $(n=971)$ : } \\
\hline ECOG 0: & $67(6.9 \%)$ \\
\hline ECOG 1: & $491(50.6 \%)$ \\
\hline ECOG 2: & $272(28.0 \%)$ \\
\hline ECOG 3: & $114(11.7 \%)$ \\
\hline ECOG 4: & $27(2.8 \%)$ \\
\hline \multicolumn{2}{|l|}{ KPS Score $(n=1017)$ : } \\
\hline KPS < 70: & $232(22.8 \%)$ \\
\hline KPS >/ 70: & $785(77.2 \%)$ \\
\hline \multicolumn{2}{|c|}{ Definitive Diagnostic Study $(n=1289)$ : } \\
\hline Bronchoscopy: & $678(52.6 \%)$ \\
\hline CT-Guided Lung Biopsy: & $297(23.0 \%)$ \\
\hline US-Guided Lung Biopsy: & $116(9.0 \%)$ \\
\hline Thoracoscopy: & $53(4.1 \%)$ \\
\hline Surgical: & $5(3.9 \%)$ \\
\hline Other': & $140(10.9 \%)$ \\
\hline \multicolumn{2}{|l|}{ Pathologic Diagnosis ( $n=1370)$ : } \\
\hline Adenocarcinoma: & $449(32.8 \%)$ \\
\hline Squamous Cell Carcinoma: & $379(27.7 \%)$ \\
\hline NSCLC-NOS: & $261(19.1 \%)$ \\
\hline Small Cell Carcinoma: & $243(17.7 \%)$ \\
\hline Adeno-Squamous Carcinoma: & $6(0.4 \%)$ \\
\hline Mesothelioma: & $5(0.4 \%)$ \\
\hline
\end{tabular}

Table 1 Patient demographics (Continued)

\begin{tabular}{ll}
\hline Neuroendocrine Tumor: & $9(0.7 \%)$ \\
Adenoid cystic Carcinoma: & $10(0.8 \%)$ \\
Others: & $8(0.6 \%)$ \\
NSCLC Stage at Diagnosis $(n=1026):$ & \\
Stage 1: & $14(1.4 \%)$ \\
Stage 2: & $42(4.1 \%)$ \\
Stage 3: & $309(30.1 \%)$ \\
Stage 4: & $661(64.4 \%)$ \\
SCLC Stage at Diagnosis $(n=220):$ & \\
Limited: & $54(24.5 \%)$ \\
Extensive: & $166(75.5 \%)$ \\
\hline
\end{tabular}

All values expressed as mean $+/-$ standard deviation, median [Inter-quartile range], or number (\%)

ECOG Eastern Cooperative Oncology Group, KPS Karnofsky Performance Scale, US ultrasound, CT computed tomography, NSCLC-NOS non-small cell lung cancer - not otherwise specified, SCLC small cell lung cancer

${ }^{\mathrm{a}}$ Income reported in rupees per month. Approximately $68.9 \mathrm{Rs}$. is equivalent to $\$ 1.00$ US

${ }^{\mathrm{b}}$ Smoking index is the number of cigarettes / bidis smoked per day multiplied by the number of years smoking (bidis are a form of tobacoco wrapped in tendu leaves and smoked like cigarettes, common in Northern India)

'Alternative procedures to obtain sample include pleural fluid analysis, sputum cytology, surface lymph node aspiration, or biopsy of a distant metastasis

presentation with a lung cancer specialist and chemotherapy initiation $(p=0.029)$.

\section{Univariate analyses of baseline characteristics}

The univariate associations between baseline patient and tumor characteristics and evaluation, treatment, and total durations are conveyed in Table 3. Patients who were diagnosed with small cell lung cancer (SCLC) required less time to receive chemotherapy after initially developing symptoms compared to those who were diagnosed with non-small cell lung cancer (NSCLC) (94.5 [65-157.3] days vs. 133 [90-210.5] days, $p<0.0001)$. In addition, patients who were previously prescribed antituberculosis treatment (ATT) required more time to undergo definitive diagnostic sampling compared to those who were not (150 [93-216] days vs. 83 [50-159] days, $p<0.0001$ ). As such, patients who were previously prescribed ATT also required more time to receive chemotherapy following the initial onset of symptoms compared to those who were not (187 [134-261.5] days vs. $113[75-180]$ days, $p<0.0001)$.

\section{Discussion}

Our retrospective analysis conveys that nearly $95 \%$ of patients with NSCLC present to our center with either stage III or VI disease while approximately $75 \%$ of patients with SCLC present with extensive-stage disease. In comparison, less than $70 \%$ of patients with NSCLC or SCLC are diagnosed with either regionally-invasive or distant metastatic disease upon presentation in the US 
Table 2 Time to presentation, diagnosis, and treatment

\begin{tabular}{|c|c|c|c|c|}
\hline & Overall & Before January 2012 & After January 2012 & $P$ value \\
\hline \multicolumn{5}{|l|}{ Evaluation Durations ${ }^{\mathrm{a}}$ : } \\
\hline Symptom Onset to Diagnostic Study $(n=755)$ : & $101[56-168]$ & $108[64-189.8]$ & $99[55-167]$ & $p=0.147$ \\
\hline Diagnostic Study to Confirmed Diagnosis $(n=908)$ : & $6[3-8]$ & $3[2-6]$ & $6[4-9]$ & $p<0.0001$ \\
\hline Symptoms Onset to Confirmed Diagnosis $(n=948)$ : & $107[60-173]$ & $114[63-192]$ & $106[59-173]$ & $p=0.194$ \\
\hline Symptom Onset to Referral with Specialist at Our Center $(n=1155)^{\mathrm{b}}$ : & $120[75-180]$ & $120[75.7-210]$ & $120[74-180]$ & $p=0.130$ \\
\hline \multicolumn{5}{|l|}{ Treatment Durations: } \\
\hline Diagnostic Study to Chemotherapy Initiation $(n=321)$ & 28 [18-42] & $25.5[13.5-35.3]$ & 28 [18-43] & $p=0.028$ \\
\hline Confirmed Diagnosis to Chemotherapy Initiation $(n=426)$ : & 20 [11-33] & $17.5[9.5-26.8]$ & $21[12-35]$ & $p=0.018$ \\
\hline Visit with Specialist at Our Center to Chemotherapy Initiation $(n=401)$ : & $12[5-19]$ & $9[5-15.3]$ & $12[6-20]$ & $p=0.029$ \\
\hline \multicolumn{5}{|l|}{ Overview: } \\
\hline Symptom Onset to Chemotherapy Initiation $(n=420)^{+}$: & $126[85-196.8]$ & $144.5[79-245]$ & $125[85.5-193.5]$ & $p=0.359$ \\
\hline New Referrals to our Center per Month (Visits/Month) ${ }^{c}$ : & $12.6+/-9.8$ & $5.1+/-2.3$ & $18.6+/-9.4$ & $p<0.0001$ \\
\hline
\end{tabular}

${ }^{\text {aAll durations were expressed as median days [inter-quartile range] }}$

${ }^{\mathrm{b}}$ Symptoms that were reported to extend longer than 90 days at time of first visit with a specialist were rounded to the nearest 30-day interval. Patients were excluded from analysis if the duration between symptom onset and referral to our center was greater than 365 days. +Patients were excluded if the duration between symptom onset and chemotherapy initiation was greater than 730 days

${ }^{c}$ New referrals per month were expressed as mean visits per month $+/-$ standard deviation

$[2,18]$. Patients managed at our center require between 4 and 5 months to receive systemic therapy following the initial onset of their symptoms. Approximately $80 \%$ of this time is consumed before a diagnosis is rendered. Less than $20 \%$ of patients are women, which is concordant with the disproportionate use of tobacco by men in South Asia [19].

\section{Comparing Total durations among centers with varying accessibility to healthcare resources}

Few large-scale investigations have previously studied the time required to diagnose lung cancer and administer treatment following the onset of symptoms. Yurdakul et al. surveyed 17 Turkish centers that had collectively served 1016 patients between 2010 and 2011 [14]. The authors retrospectively determined evaluation durations and then further prospectively followed these patients to identify treatment durations. The average time required to receive treatment after symptoms had first developed was approximately 130 days, which is similar to the median total duration that we had calculated for our retrospective cohort. Nearly all patients evaluated in their study were receiving social security and likely had better access to healthcare services than our selected patients. Redaniel et al. evaluated a cohort of nearly 6000 patients who were prospectively followed by primary care providers in the United Kingdom between 1998 and 2009 [15]. The authors observed that patients required a median interval of nearly 90 days to be diagnosed with lung cancer after developing symptoms. As this cohort was designed to study the efficiencies of ambulatory care, and patients were not followed after referral to specialists, this investigation did not report treatment durations.
Interestingly, the time needed to obtain a diagnosis of lung cancer following the initial onset of symptoms was within similar range to the interval determined for our cohort of patients. Lastly, Radzikowska et al. evaluated over 10,000 Polish patients with NSCLC between 1995 and 1998 using a national registry and found a median total duration of approximately 90 days between the onset of symptoms and administration of treatment [16]. Similar to our work and that of Yurdakul et al., the patients included in the publication by Radzikowska et al. completed questionnaires at the time of first evaluation by a lung cancer specialist to estimate the duration of their symptoms $[14,16]$. Contrarily, the work by Redaniel et al. included patients who were prospectively followed during routine visits with primary care providers [15].

As evidenced by the above, our cohort, which predominantly includes lower-income patients, shares similar total durations with cohorts of higher socioeconomic standing. Three important caveats should be noted. First, patients managed for NSCLC at our center were diagnosed with substantially higher rates of stage III and IV disease at time of presentation. As such, our patients were likely underestimating the duration of their symptoms upon evaluation at AIIMS. Consequently, evaluation durations could be greater in time, but treatment durations would remain unaffected. Second, AIIMS is a well-funded tertiary referral center. Other public hospitals in the developing world may not share similar accessibility to the necessary resources to minimize treatment delays. Therefore, generalizing our findings to other centers serving similar populations of patients may be inappropriate. Third, our work is more reflective of the current era while the aforementioned studies were 


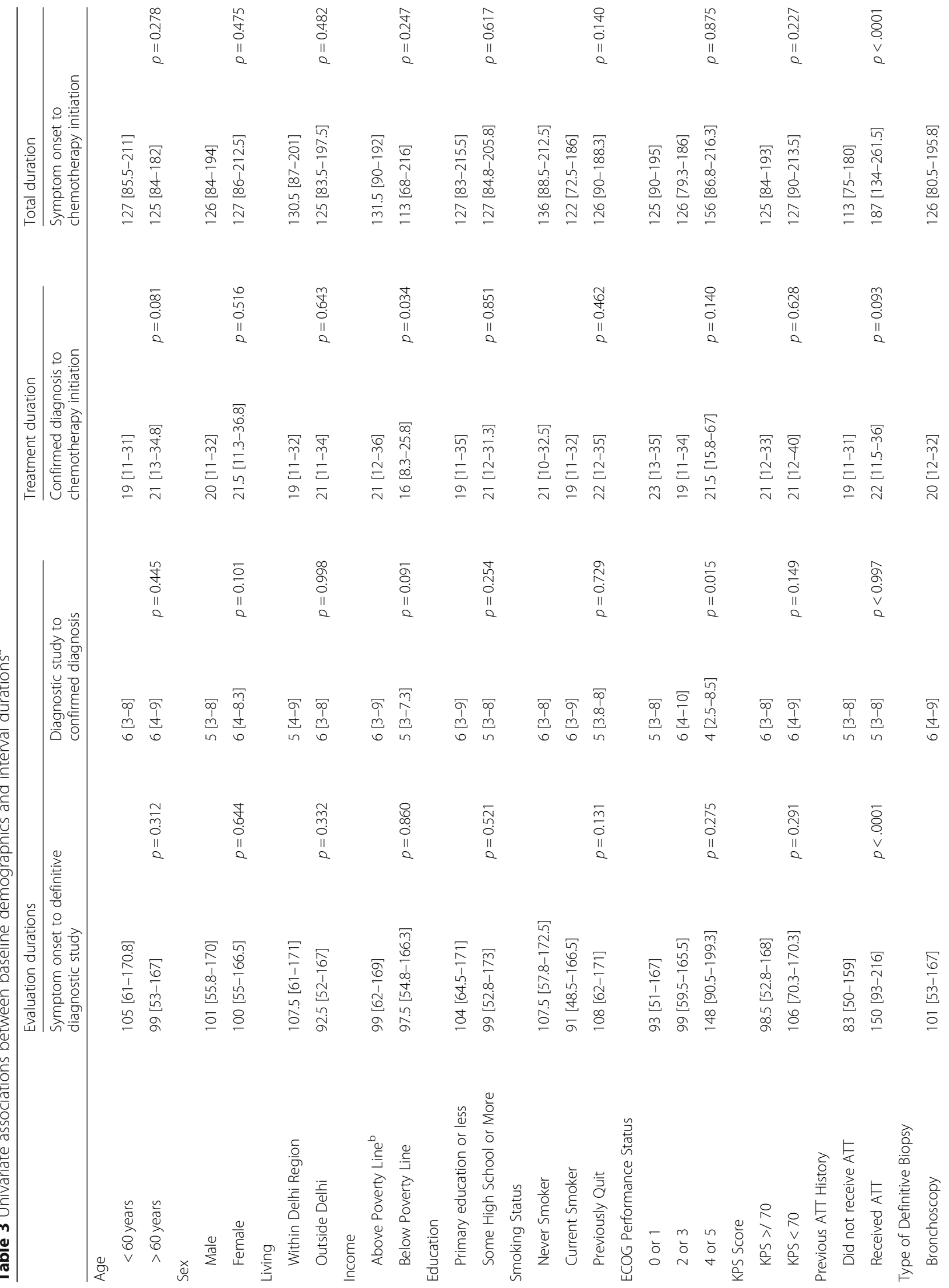




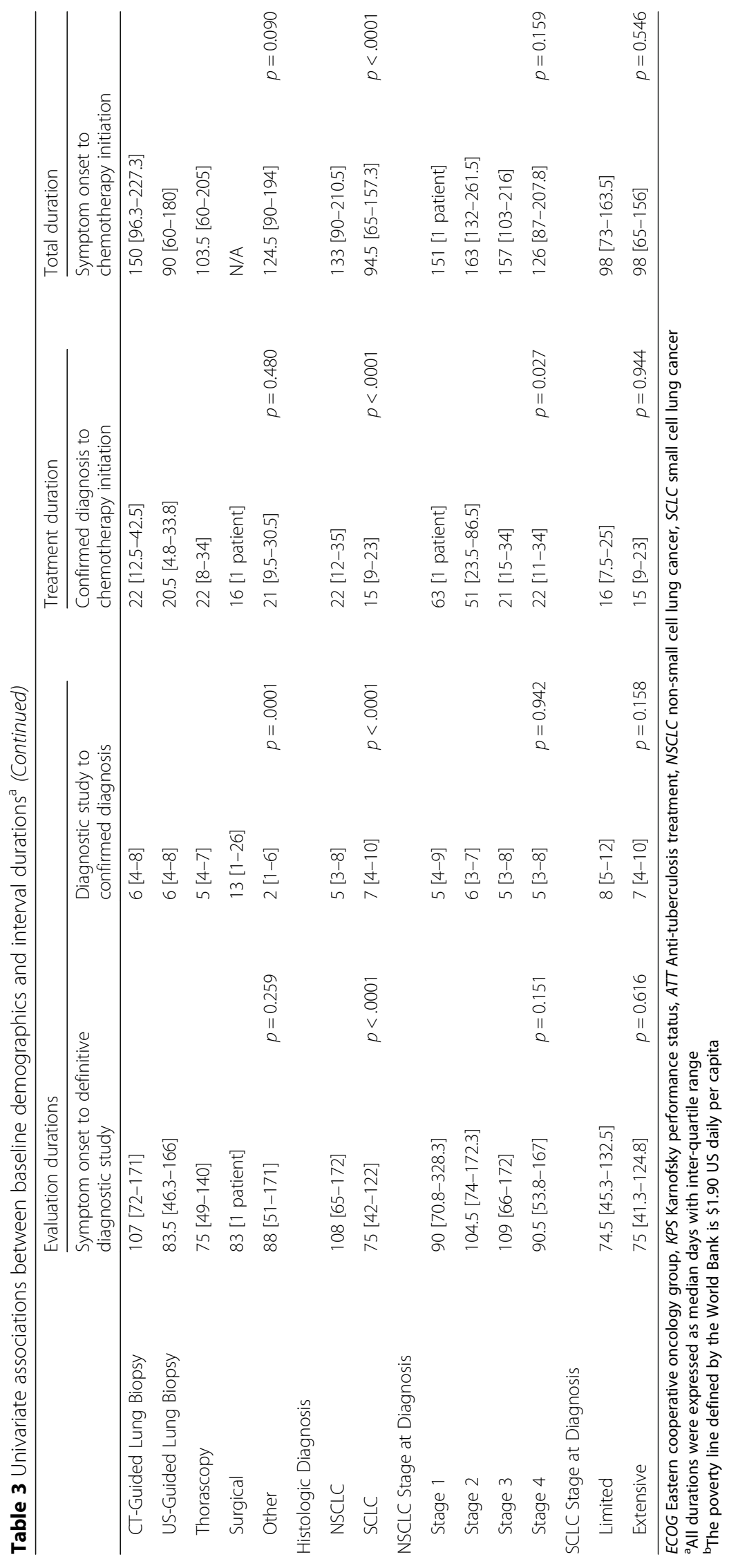


among patients studied at least 8 years ago. Patients who are now treated at those centers may require less time to obtain a diagnosis and receive treatment compared to the previously reported data.

\section{The factors that considerably prolong the diagnosis of lung cancer}

Our results indicate that the most egregious delays occur for patients during the evaluation phase. Specifically, our patients require at least 100 days to undergo a definitive diagnostic study after developing symptoms. This time interval encompasses barriers at both the patient-level and physician-level. Previous work in the United Kingdom has indicated that delays at the patient-level are often related to one of two critical issues [20, 21]. First, patients may simply be ambivalent to their condition and fail to report new symptoms to their primary care providers. Second, patients may lack access to the care required to be evaluated in a timely fashion. In regard to the first issue, we did not ask patients to detail the reasons for their postponed initial presentation to any provider upon establishing care at our center. As such, we could not determine if ambivalence was a critical factor contributing to delayed diagnoses among our patients. In regard to the second issue, we did not find any differences in the time needed to diagnose lung cancer based on level of poverty or residential location. Nonetheless, a large number of our patients were financially disadvantaged, and consequently, increasing level of poverty likely contributed minimally towards further limiting access to care.

Diagnostic delays attributed to physicians are commonly secondary to reliance on misdiagnoses. Among our cohort of patients, a reliance on tuberculosis as an alternative etiology for symptoms increased the time to diagnose lung cancer by nearly two-fold. Local physicians are reasonably concerned for tuberculosis as India unfortunately has the highest incidence of tuberculosis worldwide. Approximately one-quarter of all new diagnoses of tuberculosis are in India. Given that the presenting symptoms and initial radiographic results are similar between patients with lung cancer and those with tuberculosis, providers likely assume that the constellation of a patient's findings are consistent with tuberculosis due to the widespread prevalence of the infection [22]. Ramachandran et al. previously completed a detailed investigation of diagnostic delays by retrospectively reviewing the medical records of 96 patients who were diagnosed with lung cancer at a public referral center in southern India [23]. The authors observed that less than one-half of physicians initially suspected lung cancer based on presenting symptoms. Approximately 20\% of physicians suspected tuberculosis upon first presentation. Collectively, our findings and the results by
Ramachandran et al. convey that higher local rates of infectious diseases greatly attribute to the delays that postpone the diagnosis of lung cancer for south Asian patients. Directing efforts to increase awareness of lung cancer as a potential culprit for presenting symptoms may shorten evaluation durations and possibly reduce the incidence of metastatic disease among underserved patients.

\section{Balancing the efficient delivery of treatment with increasing volume of patients}

In regard to treatment durations, patients under care at our center were administered chemotherapy approximately 2-3 weeks following confirmed diagnoses. Despite the development of a specialty LCC, which increased the number of oncology nurses and staff, treatment was delayed by approximately 3 more days following January 2012. Most likely, this paradoxical increase in time could be attributed to a marked growth in volume of patients. The number of referrals to our center grew nearly fourfold per month after the LCC was established. Regardless, the time required to initiate treatment among our cohort of lower-income patients is in general accord with patients served within settings of higher socioeconomic status. Evans et al. previously investigated treatment durations for approximately 1400 Australian patients between 2011 and 2014 [24]. The authors observed that patients received therapy approximately 30 days following diagnoses. Contrary to our cohort, a substantial number of patients underwent surgery and/or radiation therapy. Designing and executing a multidisciplinary treatment plan requires greater collaborative efforts between physicians and staff, thereby extending the time required to initiate treatment. Shao et al. investigated treatment durations for patients receiving care at a well-funded tertiary cancer center in China and observed that approximately 2 months were required to initiate treatment [25]. Similarly, the patients reviewed by Shao et al. differ from our cohort as nearly $50 \%$ underwent radiation therapy. Nevertheless, in comparison to the aforementioned studies, our findings convey that chemotherapy is administered in a timely fashion at our center, which serves predominantly lower-income patients. The factors that postpone the management of lung cancer among our patients are largely attributable to evaluation delays at the patient-level and physician-level.

\section{Conclusion}

Based upon our single-center evaluation, a vast majority of lower-income Indian patients are staged with advanced disease at time of diagnosis. Patients require between 4 and 5 months to be diagnosed and treated with chemotherapy. Physicians contribute to diagnostic delays by attributing symptoms to tuberculosis and prescribing unnecessary ATT. Despite the development of specialty LCC, the time required to diagnose and treat lung 
cancer has increased, most likely due to a rapid increase in patient attendance. Nevertheless, the time needed to initiate therapy following diagnosis is similar between our cohort of patients and those of higher socioeconomic standing. Efforts should be directed toward encouraging physicians to maintain a high index of clinical suspicion for those at risk for lung cancer and educating patients to report concerning symptoms as early as possible.

\section{Abbreviations \\ AllMS: All India Institute of Medical Sciences; ATT: Anti-Tuberculosis Treatment; LCC: Lung Cancer Clinic; NSCLC: Non-Small Cell Lung Cancer; SCLC: Small Cell Lung Cancer}

\section{Acknowledgements}

Not Applicable.

\section{Authors' contributions}

W had full access to the data in the study and takes responsibility for the integrity of the data and the accuracy of the data analysis. CC, AG, AG2, GP $\mathrm{DJ}, \mathrm{KM}, \mathrm{VH}, \mathrm{GK}, \mathrm{RG}$, and $\mathrm{AM}$ contributed to the study design, data analysis and interpretation, and the writing of the manuscript. All authors approved the final version of this manuscript.

\section{Funding}

The authors would like to thank the Graduate Medical Office of the Cleveland Clinic Foundation for their contributions to this investigation through the Dr. William and Roxanna Michener Grant Award in Leadership Excellence, Medical Humanities, and Arts. Funding was directed towards travel and data collection.

\section{Availability of data and materials}

Data was accessed and collected from medical records within The AllMS Department of Pulmonary Medicine and Sleep Disorders.

\section{Ethics approval}

This study was formally approved by the AllMS Institutional Ethics Committee.

\section{Consent for publication}

Not Applicable.

\section{Competing interests}

The authors declare that they have no competing interests.

\section{Author details}

'Department of Medicine, Divisions of Hematology, Medical Oncology, and Hematologic Malignancies, Duke University Health System, Durham, NC, USA. ${ }^{2}$ Department of Pulmonary Medicine and Sleep Disorders, All India Institute of Medical Sciences, Delhi, India. ${ }^{3}$ Department of Gastroenterology, Mayo Clinic, Rochester, MN, USA. ${ }^{4}$ Department of Pathology, All India Institute of Medical Sciences, Delhi, India.

Received: 28 May 2019 Accepted: 29 August 2019

Published online: 03 October 2019

\section{References}

1. Ferlay J, et al. Cancer incidence and mortality worldwide: sources, methods and major patterns in GLOBOCAN 2012. Int J Cancer. 2015;136(5):E359-86 (in eng).

2. Siegel RL, Miller KD, Jemal A. Cancer statistics, 2016. CA Cancer J Clin. 2016; 66(1):7-30 (in eng).

3. Aberle DR, et al. Reduced lung-cancer mortality with low-dose computed tomographic screening. N Engl J Med. 2011;365(5):395-409 (in eng).

4. Pham D, Bhandari S, Oechsli M, Pinkston CM, Kloecker GH. Lung cancer screening rates: Data from the lung cancer screening registry. 2018;36(15_suppl):6504-4.

5. Gomez DR, et al. Time to treatment as a quality metric in lung cancer: staging studies, time to treatment, and patient survival. Radiother Oncol. 2015;115(2):257-63 (in eng).
6. Radzikowska E, Roszkowski-Sliz K, Chabowski M, Glaz P. Influence of delays in diagnosis and treatment on survival in small cell lung cancer patients. Adv Exp Med Biol. 2013;788:355-62 (in eng).

7. Samson P, et al. Effects of delayed surgical resection on short-term and long-term outcomes in clinical stage I non-small cell lung Cancer. Ann Thorac Surg. 2015;99(6):1906-12 discussion 1913, (in eng).

8. Brocken $\mathrm{P}$, et al. Timeliness of lung cancer diagnosis and treatment in a rapid outpatient diagnostic program with combined 18FDG-PET and contrast enhanced CT scanning. Lung Cancer. 2012;75(3):336-41 (in eng).

9. BTS recommendations to respiratory physicians for organising the care of patients with lung cancer. The lung Cancer working Party of the British Thoracic Society Standards of care committee. Thorax. 1998;53(Suppl 1):S1-8 (in eng).

10. Selva A, Bolibar I, Torrego A, Pallares MC. Impact of a program for rapid diagnosis and treatment of lung cancer on hospital care delay and tumor stage. Tumori. 2014;100(6):e243-9 (in eng).

11. Vinas F, Ben Hassen I, Jabot L, Monnet I, Chouaid C. Delays for diagnosis and treatment of lung cancers: a systematic review. Clin Respir J. 2016;10(3): 267-71 (in eng).

12. Chandra S, Mohan A, Guleria R, Singh V, Yadav P. Delays during the diagnostic evaluation and treatment of lung cancer. Asian Pac J Cancer Prev. 2009;10(3):453-6 (in eng).

13. V. JMP(R). SAS Institute Inc. Cary. 1989-2019.

14. Yurdakul AS, et al. Patient and physician delay in the diagnosis and treatment of non-small cell lung cancer in Turkey. Cancer Epidemiol. 2015; 39(2):216-21 (in eng).

15. Redaniel MT, Martin RM, Ridd MJ, Wade J, Jeffreys M. Diagnostic intervals and its association with breast, prostate, lung and colorectal cancer survival in England: historical cohort study using the clinical practice research datalink. PLoS One. 2015;10(5):e0126608 (in eng).

16. Radzikowska E, Roszkowski-Sliz K, Glaz P. The impact of timeliness of care on survival in non-small cell lung cancer patients. Pneumonol Alergol Pol. 2012; 80(5):422-9 (in eng).

17. World bank forecasts global poverty to fall below $10 \%$ for first time; major hurdles remain in goal to end poverty by 2030. Available: www.worldbank. org. Accessed 20 July 2018.

18. Morgensztern D, Ng SH, Gao F, Govindan R. Trends in stage distribution for patients with non-small cell lung cancer: a National Cancer Database survey. J Thorac Oncol. 2010;5(1):29-33 (in eng).

19. Mishra S, et al. Trends in bidi and cigarette smoking in India from 1998 to 2015 , by age, gender and education. BMJ Glob Health. 2016; (1):e000005 (in eng).

20. Friedemann Smith C, Whitaker KL, Winstanley K, Wardle J. Smokers are less likely than non-smokers to seek help for a lung cancer 'alarm' symptom. Thorax. 2016;71(7):659-61 (in eng).

21. Maclean R, Jeffreys M, Ives A, Jones T, Verne J, Ben-Shlomo Y. Primary care characteristics and stage of cancer at diagnosis using data from the national cancer registration service, quality outcomes framework and general practice information. BMC Cancer. 2015;15:500 (in eng).

22. Global tuberculosis report 2018. Available: https://apps.who.int/iris/handle/1 0665/274453. Accessed 20 July 2018.

23. Ramachandran K, Thankagunam B, Karuppusami R, Christopher DJ. Physician related delays in the diagnosis of lung Cancer in India. J Clin Diagn Res. 2016;10(11):Oc05-oc08 (in eng).

24. Evans SM, Earnest A, Bower W, Senthuren M, McLaughlin P, Stirling R. Timeliness of lung cancer care in Victoria: a retrospective cohort study. Med J Aust. 2016;204(2):75 (in eng).

25. Shao $Q$, et al. Clinical investigation into the initial diagnosis and treatment of 1,168 lung cancer patients. Oncol Lett. 2015;9(2):563-8 (in eng).

\section{Publisher's Note}

Springer Nature remains neutral with regard to jurisdictional claims in published maps and institutional affiliations. 